Research Article

\title{
Placebo-induced pain reduction is associated with inverse network coupling at rest
}

Isabella C. Wagner ${ }^{1}$, Markus Rütgen ${ }^{1}$, Allan Hummer ${ }^{2}$, Christian Windischberger ${ }^{2}$, and Claus Lamm ${ }^{1}$

${ }^{1}$ Social, Cognitive and Affective Neuroscience Unit, Department of Basic Psychological Research and Research Methods, Faculty of Psychology, University of Vienna, Liebiggasse 5, 1010 Vienna, Austria; ${ }^{2}$ MR Centre of Excellence, Centre for Medical Physics and Biomedical Engineering, Medical University of Vienna, Währinger Gürtel 18-20, 1090 Vienna, Austria

Correspondence: Isabella C. Wagner, Social, Cognitive and Affective Neuroscience Unit, Department of Basic Psychological Research and Research Methods, Faculty of Psychology, University of Vienna, Liebiggasse 5, 1010 Vienna, Austria, Phone: +43 (0)1 4277 47133, Email: isabella.wagner@univie.ac.at 


\begin{abstract}
Placebos can reduce pain by inducing beliefs in the effectiveness of an actually inert treatment. Such topdown effects on pain typically engage lateral and medial prefrontal regions, the insula, somatosensory cortex, as well as the thalamus and brainstem during pain anticipation or perception. Considering the level of large-scale brain networks, these regions spatially align with fronto-parietal/executive control, salience, and sensory-motor networks, but it is unclear if and how placebos alter interactions between them during rest. Here, we investigated how placebo analgesia affected intrinsic network coupling. Ninety-nine human participants were randomly assigned to a placebo or control group and underwent resting-state fMRI after pain processing. Results revealed inverse coupling between sensory-motor and salience-like networks in placebo but not control participants. Specifically, networks were centered on the bilateral somatosensory cortex, as well as on the brainstem, thalamus, striatal regions, dorsal and rostral anterior cingulate cortex, and the insula, respectively. Across participants, more negative between-network coupling was associated with lower individual pain intensity as assessed during a preceding pain task, but was unrelated to expectations of medication effectiveness in the placebo group. Altogether, these findings provide initial evidence that placebo analgesia affects the intrinsic communication between large-scale brain networks, even in the absence of pain. We suggest a model where placebo analgesia increases activation within a descending pain-modulatory network, segregating it from somatosensory regions that might code for painful experiences.
\end{abstract}

\title{
Highlights
}

- Placebo analgesia affects resting-state connectivity between networks.

- Salience-related and somatosensory regions are negatively coupled at rest.

- This coupling is negative following placebo, but not in control participants.

- More negative between-network coupling is related to lower pain intensity.

\section{Keywords}

placebo analgesia| pain | resting-state fMRI | brain networks | independent component analysis (ICA) 
I.C. Wagner et al.

\section{Introduction}

Placebos can reduce pain by inducing beliefs in the effectiveness of an actually inert treatment (Price et al., 2008). This top-down effect on pain engages brain regions that signal pain anticipation, treatment expectations, and reward-processes linked to pain relief, such as the lateral prefrontal cortex (Wager et al., 2004, 2007; Watson et al., 2009; Petrovic et al., 2010), rostral anterior cingulate cortex (rACC; Petrovic et al., 2002; Zubieta et al., 2005; Eippert et al., 2009), periaqueductal grey (PAG; Petrovic et al., 2002; Wager et al., 2007), and the ventral striatum (Zubieta et al., 2005; Scott et al., 2007; Geuter et al., 2013). During placebo analgesia, these structures are thought to work in concert with regions associated with actual pain perception, including the thalamus, insula, dorsal anterior cingulate (dACC) and somatosensory cortices (Zubieta et al., 2001; Petrovic et al., 2002; Wiech et al., 2010; Bingel et al., 2011). Previous studies demonstrated enhanced placebo-related coupling between the rACC and PAG (Petrovic et al., 2002; Bingel et al., 2006; Wager et al., 2007; Eippert et al., 2009), as well as interactions between the lateral prefrontal cortex, thalamus, and insula (Lorenz et al., 2003; Wager et al., 2007). Thus, connectivity between regions related to pain processing appears to play a role in placebo-induced pain reduction.

The set of regions involved in placebo analgesia spatially aligns with resting-state networks such as the fronto-parietal/executive control and salience networks (Dosenbach et al., 2007; Seeley et al., 2007), as well as with the sensory-motor network anchored on pre- and postcentral gyri, supplemental motor area, and posterior insula (Beckmann et al., 2005; Damoiseaux et al., 2006; Yeo et al., 2011). The fronto-parietal network (FPN, or "executive control network", ECN) comprises the lateral prefrontal cortex and inferior parietal regions (Vincent et al., 2008; Yeo et al., 2011) and is relevant for cognitive control (Dosenbach et al., 2006, 2007; Cole et al., 2013). The so-called "salience network" (SN; Seeley et al., 2007) is a sub-system of the FPN centered on the anterior mid-cingulate cortex (or dorsal anterior cingulate cortex, dACC) and insula, and is implicated in attention (Downar et al., 2000), negative affective processing (Seeley et al., 2007) and pain perception (Downar et al., 2003; Legrain et al., 2011; Wager et al., 2013; Kucyi and Davis, 2015). Kong and colleagues (2013), for example, demonstrated resting-state functional connectivity between the FPN/ECN and the rACC which was related to pain modulation in a subsequent task (Kong et al., 2013). Tétreault and colleagues (2016) used resting-state at the beginning of a clinical trial with chronic knee osteoarthritis pain patients to identify placebo responders (Tétreault et al., 2016). They found increased whole-brain coupling of the right midfrontal gyrus that predicted pain relief after placebo treatment. Similarly, Sikora and colleagues (2016) found enhanced connectivity between the rACC and the $\mathrm{SN}$ at baseline that was associated with greater symptom reduction after placebo treatment in patients with major depressive disorder (Sikora et al., 2016). Thus, resting-state connectivity involving regions of the FPN/ECN and the SN seems to predict individual placebo responses at baseline, prior to placebo treatment, in different patient populations. Crucially, however, how placebos alter intrinsic network interactions in healthy humans is so far unclear. Therefore, we investigated the effects of placebo analgesia on network interactions at rest within a large-scale, healthy participant sample.

Ninety-nine participants were randomly assigned to a placebo or control group and underwent functional MRI (see also Rütgen et al., 2015). Neural activity during a rest period that completed the scan session was compared between groups. To the best of our knowledge, effects of placebo analgesia on restingstate functional connectivity between networks have not yet been investigated. We thus chose an exploratory and predominantly data-driven analysis approach to do so for the first time. This included the identification of common resting-state networks (Beckmann and Smith, 2004) and the quantification of functional connectivity between them (Smith et al., 2013). Based on previous findings regarding baseline connectivity changes that predicted subsequent (placebo-related) pain modulation (see above; Kong et al., 2013; Sikora et al., 2016; Tétreault et al., 2016), we hypothesized changes in connectivity between the FPN/ECN and SN in placebo compared to control participants, as well as interactions with the sensory- 
motor network. Additionally, we reasoned that connectivity changes should scale with a behavioral pain intensity score that captured placebo-induced pain relief during a preceding task. Results might shed first light on how placebo analgesia affects whole-brain, intrinsic network fluctuations at rest.

\section{Materials and methods}

\subsection{Participants}

One-hundred-and-twenty participants were randomly assigned to a placebo ( $N=60,43$ females) or control group ( $N=60,38$ females). Eighteen participants had to be excluded due to non-responding to the placebo manipulation (10), or technical problems (8). This left 102 participants for the analyses reported by Rütgen and colleagues (2015; placebo group: $N=49,36$ females, age range $=20-31$ years, mean age $=25$; control group: $N=53,34$ females, age range $=19-38$ years, mean $=26)$. From this sample, we had to exclude 3 participants (placebo group, 1; control group, 2) due to technical problems with the resting-state data recordings. The current analyses thus included 99 participants (placebo group: $N=48$, 35 females, age range $=20-31$ years, mean $=24$; control group: $N=51,34$ females, age range $=19-38$ years, mean $=25$ ). All were right-handed, healthy, had normal or corrected-to-normal vision, and gave written informed consent prior to participation. The study was reviewed and approved by the ethics committee of the Medical University of Vienna (Vienna, Austria).

\subsection{Study setup and experimental design}

This study was part of a larger project investigating the effects of placebo analgesia on pain and pain empathy (Rütgen et al., 2015). In brief, participants completed a pain calibration procedure upon arrival in the laboratory. After this, the placebo group underwent placebo induction consisting of pill intake and conditioning. Participants then entered the MR scanner and completed a pain, as well as an affective touch task (reported elsewhere), followed by the structural scan. The session was concluded with a resting-state period (Fig. 1AB).

\subsubsection{Electrical stimulation and pain calibration}

Individual intensity values $(\mathrm{mA})$ for electrical stimulation were determined during pain calibration (15-30 min). This involved a staircase procedure where participants were asked to rate pain intensity after every electrical shock (500 ms), using a 7-point scale (1, "perceptible, but no painful sensation"; 7, "extreme pain"). The same scale was used for pain intensity ratings throughout the study. Electrical stimulation was delivered using a Digitimer DS5 Isolated Bipolar Constant Current Stimulator (Digitimer Ltd, Clinical \& Biomedical Research Instruments) and a bipolar concentric surface electrode attached to the dorsum of the left hand. Shock delivery was controlled manually using Cogent (version 1.32, www.vislab.ucl.ac.uk/cogent.php).

\subsubsection{Placebo induction: pill intake and conditioning}

Participants of the placebo group were introduced to a medical doctor who administered the placebo pill (starch pill, prepared by the institutional pharmacy of the Medical University of Vienna) and gave information about the alleged medication (see Rütgen et al., 2015 for details). Participants were then asked to rate the expected effectiveness ("Do you expect this medication to be effective in reducing your pain?"; effectiveness ${ }_{T_{1}}$ ) on a scale from 1 ("not at all") to 7 ("very effective"). This was followed by a waiting time (15 $\mathrm{min}$ ) for the alleged medication "to take effect", after which classical conditioning was used to strengthen the placebo analgesic response (10-15 min; see Rütgen et al., 2015). After this, participants were once more asked how effective they thought the medication was in reducing pain ("How 
effective is this medication?"; effectiveness T2 $_{2}$. The control group did not undergo placebo induction and entered the MR scanner after the initial pain calibration (Fig. 1A).

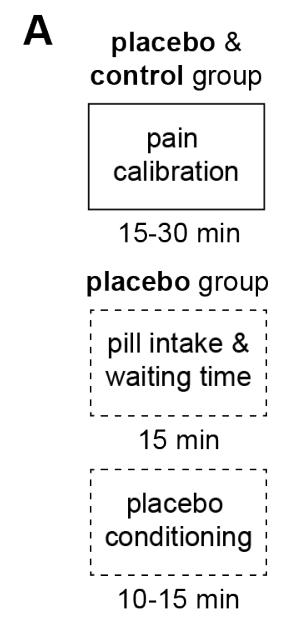

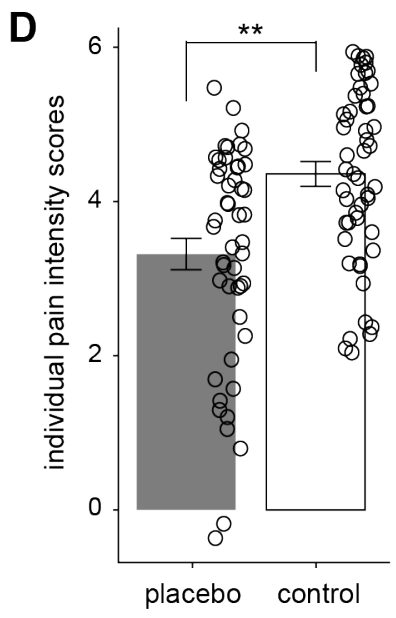

B

MRI
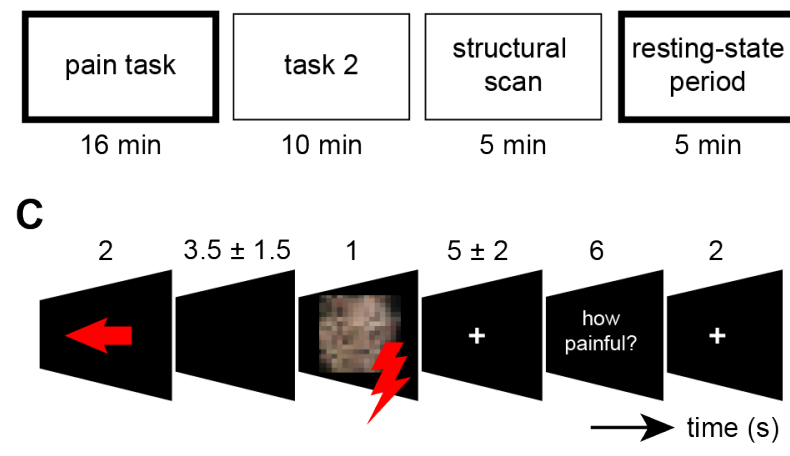

Fig. 1. Study timeline, pain task, and individual pain intensity scores. (A) Participants of placebo and control groups completed the pain calibration procedure. For the placebo group, this was followed by pill intake, waiting time, and placebo conditioning (dashed framing). (B) Tasks and scanning periods that were completed within the MR scanner. Participants first completed the pain task, an affective touch task (task 2, reported elsewhere), the structural scan, and a resting-state period. Bold framing indicates the data used to calculate individual pain intensity scores (pain task; Section 2.2.4.), and the data used for the imaging analyses (resting-state period). (C) Example for a selfdirected, painful trial during the pain task. (D) Distribution of individual pain intensity scores in both groups. ${ }^{* *}$ indicates significantly lower pain intensity scores in the placebo compared to the control group, $p<0.001$.

\subsubsection{Pain task}

Inside the MR scanner, participants completed the pain task (16 min), an affective touch task ( $2 \times 5 \mathrm{~min}$, reported elsewhere), and a structural scan (5 min; Fig 1B). During the pain task, participants received a cue $(2 \mathrm{~s})$ if the electrical shock was directed at themselves (arrow pointing left, self-directed trial) or at another participant (arrow pointing right, other-directed trial). Additionally, the color of the arrow informed the participant about the upcoming stimulation intensity (red, painful; green, non-painful). The other participant was actually a member of the experimental team and never received any shocks. After a brief delay between 2 and $5 \mathrm{~s}$ (mean $=3.5 \mathrm{~s}$ ), participants saw a photo of the shock recipient on the screen ( $1 \mathrm{~s}$; self-directed trial, scrambled photo of themselves; other-directed trial, photo of the confederate with painful/non-painful facial expression), and a brief electrical shock (500 ms) was 
delivered (during self-directed trials only). After another fixation period ranging from 3 to $7 \mathrm{~s}$ (mean $=5$ s), affect ratings ( $6 \mathrm{~s}$ ) were collected during one-third of the trials (self-directed pain ratings: "How painful was this stimulus for you?", other-directed affect ratings: "How painful was this stimulus for the other person?", and "How unpleasant did it feel when the other person was stimulated?"). Trials were separated with a short fixation period ( $2 \mathrm{~s}$ ). In total, participants completed 15 trials per condition (i.e., self-directed painful, self-directed non-painful, other-directed painful, other-directed non-painful). Here, we will focus on self-directed painful and non-painful trials only. An example for a self-directed painful trial is given in Fig. 1C. The task was programmed and presented with Cogent (version 1.32, www.vislab.ucl.ac.uk/cogent.php).

Stimulation intensities during self-directed trials for both placebo and control groups were set to calibration-specific stimulation intensities related to pain intensity ratings of 1 (i.e., non-painful trial) or 7 (i.e., extremely painful trial) throughout the pain task. The average stimulation intensities during the pain task were $0.16 \pm 0.14 \mathrm{~mA}$ (mean $\pm \mathrm{SEM}$; pain rating of 1 ) and $0.74 \pm 0.6 \mathrm{~mA}$ (pain rating of 6 ) during nonpainful and painful trials, respectively. Stimulation intensities during non-painful (placebo group: $0.13 \pm$ $0.34 \mathrm{~mA}$, control group: $0.17 \pm 0.44 \mathrm{~mA}: t(92.86)=1.148, p=0.254$ ) and painful trials (placebo group: 0.63 $\pm 1.36 \mathrm{~mA}$, control group: $0.85 \pm 1.91 \mathrm{~mA}: t(89.09)=1.874, p=0.064)$ did not differ significantly between groups.

\subsubsection{Individual pain intensity scores}

We aimed at identifying placebo effects on whole-brain resting-state connectivity. Specifically, we reasoned that placebo effects on between-network connectivity would be related to changes in individual pain intensity ratings. To this end, we leveraged data from the pain task (see above) and calculated a behavioral "pain intensity score" for each participant. We subtracted individual pain intensity during nonpainful trials from pain intensity during painful trials (painful minus non-painful), thereby normalizing individual pain intensity scores for general stimulation intensity. These scores were then correlated with results from the between-network connectivity analysis (Section 2.4.3.).

\subsubsection{Resting-state period}

To assess intrinsic connectivity changes related to placebo analgesic effects, the MR session was concluded with a 5-minute resting-state period. Participants were instructed to remain awake with their eyes open while a white fixation cross was presented at the center of the computer screen. For the placebo group, the resting-state period took place approximately $30 \mathrm{~min}$ after the placebo induction (Section 2.2.2.). For the control group, the resting-state period took place approximately $30 \mathrm{~min}$ after the pain calibration (Fig. 1AB).

\subsection{MRI data acquisition}

Imaging data were acquired using a 3 Tesla MRI scanner (Tim Trio, Siemens, Erlangen, Germany) equipped with a 32-channel head coil. We obtained $200 \mathrm{~T}_{2}{ }^{*}$-weighted BOLD images during the resting-state period, using a multiband-accelerated echoplanar imaging (EPI) sequence. Parameters were as follows: TR $=1800$ $\mathrm{ms}, \mathrm{TE}=33 \mathrm{~ms}$, flip angle $=60^{\circ}$, interleaved slice acquisition, 54 axial slices, FOV $=192 \times 192 \times 108 \mathrm{~mm}$, matrix size $=128 \times 128$, voxel size $=1.5 \times 1.5 \times 2 \mathrm{~mm}$. Structural scans were acquired using a magnetization-prepared rapid gradient echo (MP-RAGE) sequence with the following parameters: $T R=$ $2300 \mathrm{~ms}, \mathrm{TE}=4.21 \mathrm{~ms}, 160$ sagittal slices, FOV $=256 \times 256 \mathrm{~mm}$, voxel size $=1 \times 1 \times 1.1 \mathrm{~mm}$. 
I.C. Wagner et al.

\subsection{Data processing and statistical analysis}

\subsubsection{MRI data preprocessing}

All imaging data were analyzed using the Functional Magnetic Resonance Imaging of the Brain (FMRIB) Software Library (FSL, v5.0.1; https://fsl.fmrib.ox.ac.uk/fsl/fslwiki/; Jenkinson et al., 2012). As a first step, the structural scan was processed (using $f s$ _anat), including the re-orientation to the Montreal Neurological Institute (MNI) standard space (fs/reorient2std), bias-field correction (FAST), and brain extraction (BET). The functional images were preprocessed using FEAT. We excluded the first eight volumes to account for T1 equilibration, performed motion correction (MCFLIRT), spatial smoothing with a Gaussian kernel (5 $\mathrm{mm}$ full-width at half maximum), and aligned images to the bias-corrected, brainextracted structural image (FLIRT) using boundary-based registration. The structural image was aligned with the MNI 152 EPI template using non-linear registration (FNIRT). After manual inspection of the registered images, we used independent component analysis (ICA) to automatically detect and remove participant-specific, motion-related artifacts (ICA-based strategy for Automatic Removal Of Motion Artifacts, ICA-AROMA, v0.3-beta; Pruim et al., 2015a, 2015b). Following data de-noising, we applied the registration parameters to the functional data (using applywarp).

\subsubsection{Group Independent Component Analysis (Group-ICA)}

The de-noised functional data were high-pass filtered with a cutoff at $0.01 \mathrm{~Hz}$. We did not apply bandpass filtering as it was shown that high frequencies in the BOLD signal can carry relevant information (Shirer et al., 2015). Data from both placebo and control groups were then submitted to a group-ICA using MELODIC (Beckmann and Smith, 2004). This procedure concatenated all 99 four-dimensional participant data sets into one time $\times$ voxel $\times$ participant matrix and segregated them into independent spatiotemporal components. The maximum number of independent components (ICs) was set to 30 as this was shown to yield the most common resting-state networks (Abou-Elseoud et al., 2009).

Next, we used a novel, combined approach to separate ICs corresponding to intrinsic resting-state networks from those reflecting noise. We evaluated the $30 \mathrm{ICs}$ in two steps that consisted of manual evaluation (step 1) and an automatic template-matching procedure (step 2). By comparing the ICs to two different, well-known resting-state parcellations (Beckmann et al., 2005; Yeo et al., 2011), we were able to assign the ICs to resting-state networks independent of parcellation-specific network nomenclature.

During step 1, we manually compared each IC to the eight most common resting-state networks as reported by Beckmann and colleagues (2005). This included the (1) medial visual cortical network, (2) lateral visual cortical network, (3) auditory network, (4) sensory-motor network, (5) default-mode network, (6) executive control network, (7) left-, and (8) right dorsal visual networks. Of the 30 ICs, 15 ICs could clearly be assigned to one of the above networks (classified as "signal"), 7 ICs resembled signalrelated network fluctuations that could not clearly be assigned to one of the above networks (classified as "unclear/signal"; Griffanti et al., 2017), and the remaining 8 ICs were manually classified as noiserelated ("noise").

During step 2, we used an automatic template-matching procedure (using spatial cross-correlations, fslcc; minimum correlation, $r=0.2$ ) to identify ICs that resembled the seven most common resting-state networks as defined by Yeo and colleagues (2011; spatial maps were downloaded from https://surfer.nmr.mgh.harvard.edu/fswiki/CorticalParcellation_Yeo2011). These networks included the (1) ventral and (2) dorsal attention networks, (3) default-mode network, (4) fronto-parietal network (FPN), (5) a network containing motor and auditory regions, (6) a network resembling the salience network (SN), and (7) a network anchored on the ventromedial prefrontal cortex and anterior-ventral temporal lobe. Of the 30 ICs, 22 ICs were assigned to one or more of the above networks (classified as "signal"). The 
remaining $8 \mathrm{ICS}$ overlapped with ICs that could not clearly be assigned to a specific resting-state network during step 1 ("unclear"). For these, we checked if the ICs were previously classified as "unclear/signal" or "noise". More specifically, if an IC could not be assigned to a network using spatial cross-correlations (step 2, "unclear") but was classified as likely being signal-related using the manual evaluation (step 1, "unclear/signal"), then this IC was included in all further analyses. If an IC could not be assigned to a network using spatial cross-correlations (step 2, "unclear") and if it was classified as noise using the manual evaluation (step 1, "noise"), then this IC was excluded from all further analyses. Overall, 5 out of 30 ICs were classified as noise-related. All remaining analyses were thus performed using a set of 25 ICs (Fig. 2). For display purposes, the ICs were brought into $2 \mathrm{~mm} \mathrm{MNI} \mathrm{standard} \mathrm{space} \mathrm{using} \mathrm{FLIRT.}$

After group-ICA, we applied dual regression to generate participant-specific spatial maps and associated time series (Smith et al., 2014) by regressing each IC's spatial map into the participants' four-dimensional space $\times$ time data set. 


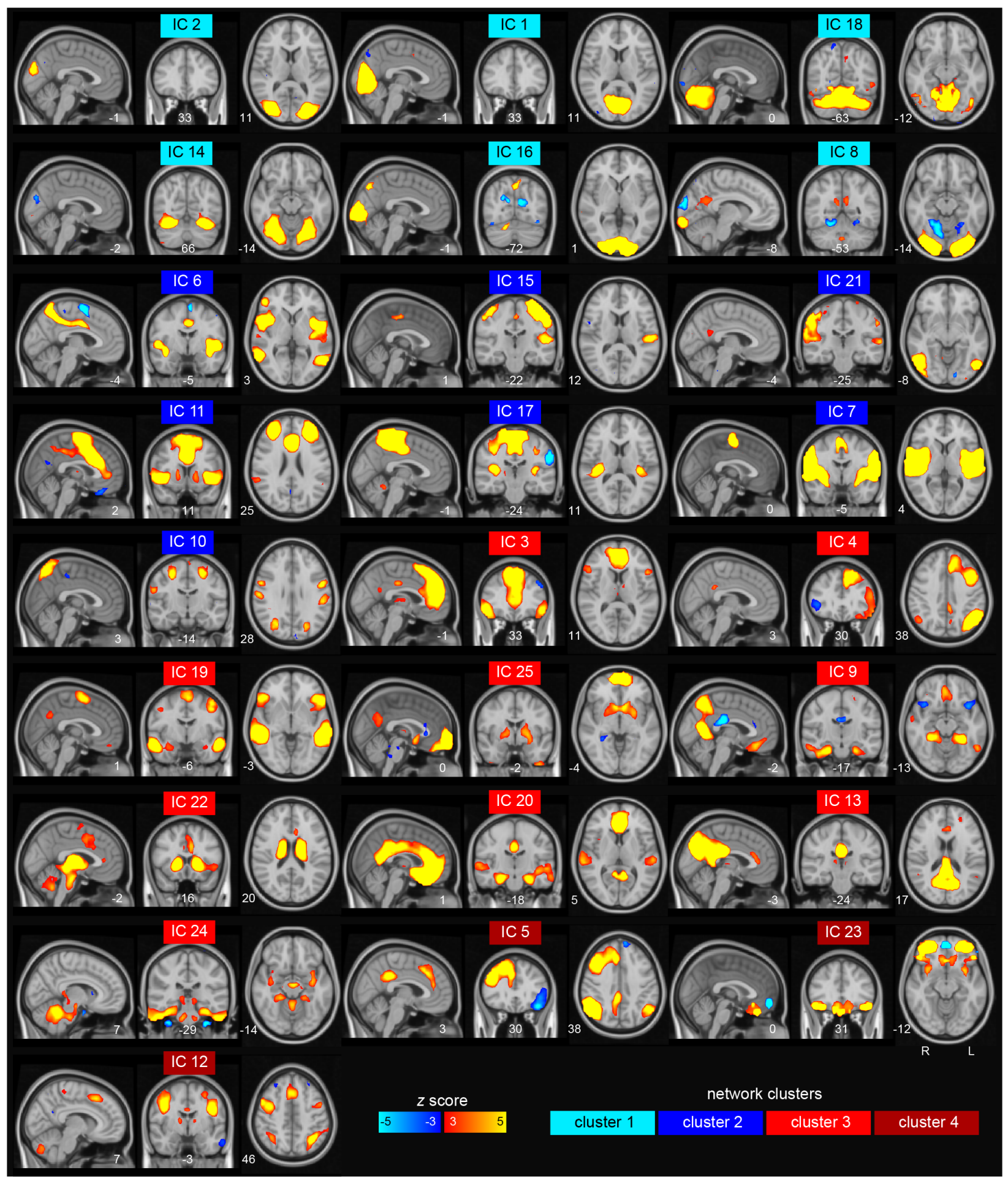

Fig. 2. Resting-state networks identified through Group-ICA. Results from group-ICA across both placebo and control groups ( $N=99$ ) yielded 25 independent components (ICs) that were used for all further analyses. ICs were grouped based on their between-network connectivity which resulted in four clusters of resting-state networks (see also Fig. 3A) centered on visual networks (cluster 1, marked in cyan), sensory-motor networks (cluster 2, marked in blue), networks resembling the DMN (cluster 3, marked in red), and networks resembling the FPN/ECN (cluster 4, marked in dark red). Note that ICs are shown according to neurological convention: R, right; L, left. 
I.C. Wagner et al.

\subsubsection{Between-network connectivity}

Resting-state connectivity between networks was determined using FSL Nets (Smith et al., 2013) and Matlab (Matlab 2017b, The Mathworks, Natick, MA, USA). The participant-specific time series resulting from dual regression (Section 2.4.2.) were used to (1) evaluate between-network connectivity across participants, and (2) to compare between-network connectivity between the placebo and control groups. More specifically, we created a $25 \times 25$ correlation matrix of all ICs (i.e., nodes) per participant where each element represented the correlation strength (i.e., edge) between two nodes. We then calculated full and partial correlation coefficients, the latter to estimate the direct connections between brain regions, and transformed them from Pearson's $r$ to Fisher's $z$-values. The participant-specific network matrices were then reshaped into a single row and combined into a participant $\times$ edges network matrix to generate the functional connectome of the whole group (i.e., mean network matrix, placebo and control). To group similar nodes, we used hierarchical clustering on the full correlations of the mean network matrix and rearranged the 25 nodes based on their spatiotemporal characteristics using Ward's method (Ward, 1963; Fig. 3A).

Group-level statistics were implemented using FSL's randomise with 5000 permutations per contrast. This tested for a significant effect at each of the 625 network edges by estimating edge-specific $p$-values, corrected for multiple comparisons across all edges. First, we determined significant between-network connectivity across participants using a one-sample $t$-test. Second, we assessed significant differences in between-network connectivity between the placebo and control groups using an independent-sample $t$ test (contrasts: placebo > control, control > placebo). Finally, to test the behavioral significance of the obtained differences in between-network connectivity between groups, we extracted the edge values and performed a cross-participant correlation analysis between individual edge connectivity and pain intensity scores (Section 2.2.4.).

\subsubsection{Scan-to-scan motion between groups}

Lastly, we assured that our connectivity results were not confounded by differences in scan-to-scan motion between the groups. We calculated the temporal derivative time course of the root mean square variance based on all voxels of each brain image (DVARS; Power et al., 2012). This gave an image-specific measure of intensity change by comparing to the previous image. The average DVARS was generally small (mean \pm SEM, placebo group: $46.3 \pm 1.1$; control group: $49.1 \pm 1.15$ ) and did not significantly differ between groups $(p=0.081)$. Thus, the amount of movement during resting-state was comparable between participants of the placebo and control groups.

\subsection{Data and code availability statement}

All anonymized data and analysis code are available upon request in accordance with the requirements of the institute, the funding body, and the institutional ethics board.

\section{Results}

\subsection{Expectations in medication effectiveness and individual pain intensity scores}

To start out, we evaluated the effectiveness of the placebo medication as rated by the placebo group before and after the conditioning procedure (effectiveness ${ }_{T 1}$, "Do you expect this medication to be effective in reducing your pain?"; effectivenesS ${ }_{T 2}$, "How effective is this medication?"; Section 2.2.2.). Ratings significantly increased from effectivenesS $\mathrm{T}_{11}$ (mean \pm standard error of the mean, SEM: $4.35 \pm 0.16$ ) to effectiveness T2 $_{2}(4.75 \pm 0.21, t(47)=-2.479, p=0.017, d=-0.31,[95 \% \mathrm{Cl},-0.72,0.1])$, indicating that the conditioning procedure increased expectations of medication effectiveness. 
To test the effects of placebo on subjective pain intensity, we compared individual pain intensity scores obtained during the pain task between the groups (i.e., pain ratings during painful minus non-painful trials; Section 2.2.4.). Pain intensity scores were significantly lower in the placebo (mean \pm SEM: $3.32 \pm$ 0.41 ) compared to the control group $(4.36 \pm 0.32, t(90.031)=4.028, p<0.0005, d=-0.82,[95 \% \mathrm{Cl},-1.23$, $-0.4]$; Fig. 1D). Thus, placebo successfully decreased subjective pain intensity with an effect size similar to previous placebo analgesia neuroimaging studies (Zunhammer et al., 2018).

\subsection{Between-network connectivity across groups}

As a first step, we identified 25 resting-state networks based on data from both placebo and control groups (Fig. 2, Section 2.4.2.). Using these, we calculated the mean connectivity between every network (i.e., node) which yielded a correlation matrix of between-network connectivity values (i.e., edges; Fig. 3A). Highly correlated nodes were grouped together during hierarchical clustering (Section 2.4.3.). This resulted in four network clusters (Fig. 2 and 3): cluster 1 contained visual networks (centered on visual cortex but also on the cerebellum; marked in cyan); cluster 2 contained sensory-motor networks (anchored on the motor, somatosensory and auditory regions, but also dACC and bilateral insula; marked in blue); cluster 3 comprised components of the default-mode network (DMN; including frontal and posterior medial regions, the hippocampus, surrounding medial temporal lobe, and parietal regions; marked in red); and cluster 4 included a right lateralized fronto-parietal/executive control network (FPN/ECN; marked in dark red).

Notably, cluster 3, which was centered on the DMN, also contained a sub-cluster that was only loosely connected to the remaining networks. This sub-cluster included a network that resembled the salience network (SN; IC 22; Fig. 2, marked in red). Another network that was spatially similar to the SN (IC 11) showed between-network connectivity with components of cluster 2 which contained sensory-motor networks (Fig. 2, marked in blue). While cluster 4 included a right lateralized FPN/ECN (IC 5; Fig. 2, marked in dark red), its left lateralized counterpart (IC 4) was mainly coupled with networks of cluster 3 . Thus, both FPN/ECN and SN revealed between-network connectivity with networks of different clusters rather than mainly coupling with networks belonging to the FPN. 
bioRxiv preprint doi: https://doi.org/10.1101/735563; this version posted August 15,2019 . The copyright holder for this preprint (which was not certified by peer review) is the author/funder, who has granted bioRxiv a license to display the preprint in perpetuity. It is made available under aCC-BY-NC-ND 4.0 International license.

I.C. Wagner et al.

A

all participants $(N=99)$

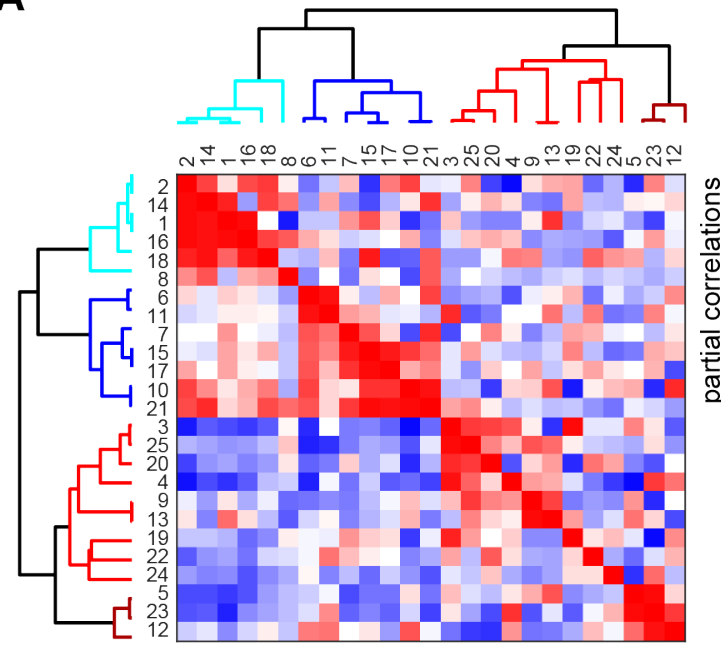

full correlations

Fisher's z

B
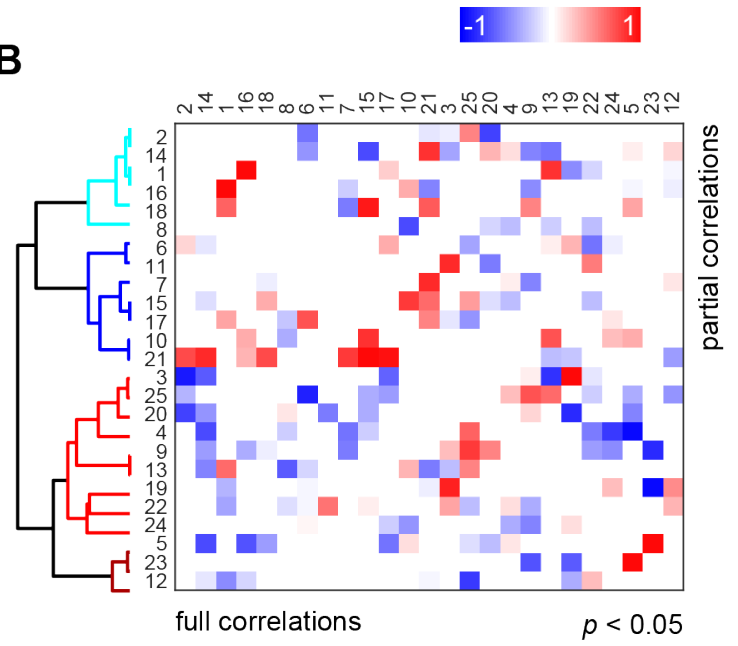

C

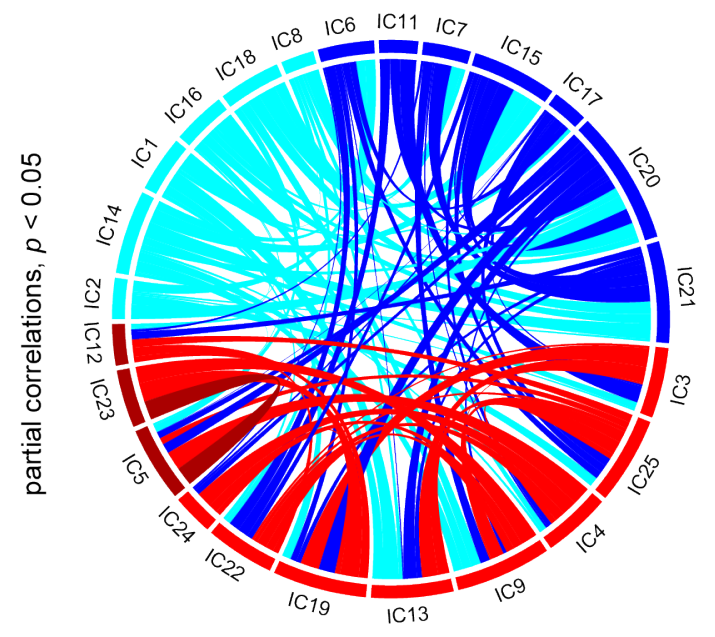

network clusters 
Fig. 3. Between-network connectivity across all participants $(N=99)$. (A) Hierarchical clustering yielded four groups of resting-state network that were clustered based on their between-network connectivity. The correlation matrix shows full (below diagonal) and partial (above diagonal) correlations between networks. Numeric labels correspond to the independent components (ICS) visible in Fig. 2. (B) Thresholded connectivity matrix showing only significant between-network connections ( $p<0.05$; corrected for multiple comparisons across all 625 network edges). Significant partial correlations (above diagonal) were used for all further analyses. (C) Chord diagram visualizing significant, direct between-network connections across all participants.

\subsection{Group differences in between-network connectivity}

Most importantly, we hypothesized differences in between-network connectivity between the placebo and control groups. We expected that these connectivity changes should involve the FPN/ECN and SN, as well as interactions with the sensory-motor network in placebo compared to control participants.

Result revealed connectivity between a network assigned to cluster 2 (centered on sensory-motor networks; IC 15) and a network assigned to cluster 3 (centered on the DMN; IC 22) that differed significantly between the placebo and control groups (control > placebo; $p=0.047$, corrected for multiple comparisons across all 625 network edges; Fig. 4A). These networks were negatively correlated in placebo participants (one-sample $t$-test against zero: $t(47)=-5.281, p<0.0005$ ) and not significantly correlated in participants of the control group ( $p=0.761$; Fig. 4B). In other words, IC 15 and 22 were inversely coupled in the placebo compared to the control group such that decreased activation within IC 15 was associated with increased activation within IC 22 and vice versa. IC 15 comprised a network including the bilateral somatosensory cortex, supplemental motor area, posterior insula/left superior temporal gyrus, and the cerebellum (Fig. 4C, upper panel; Table 1). IC 22 consisted of a network between the cerebellum and brainstem, bilateral thalamus and striatal regions, dorsal and rostral ACC, and the anterior insula (Fig. 4C, lower panel; Table 1). There were no other significant group-differences in between-network connectivity.

\subsection{Between-network connectivity and relation to individual pain intensity scores}

So far, we reported lower pain intensity scores and inverse coupling between two resting-state networks (IC 15-22) in placebo compared to control participants. As a final step, we tested the cross-participant relationship between behavioral pain intensity scores and between-network connectivity using correlation analysis. Results demonstrated a significantly positive correlation of between-network connectivity (IC 15-22) and individual pain intensity scores across all participants $(r=0.253, p=0.011$; bootstrapped $95 \% \mathrm{Cl}$ based on 5000 samples $=[0.08,0.44]$ ), that remained stable after the exclusion of two possible outliers (placebo group; $r=0.218, p=0.032$, bootstrapped 95\% Cl [0.03, 0.41]; Fig. 4D). Altogether, this indicates that greater inverse coupling between networks that were anchored on the bilateral somatosensory cortex (IC 15), as well as on the brainstem, thalamus, striatal regions, dorsal and rostral ACC, and the anterior insula (IC 22) was related to lower subjective pain intensity during the preceding pain task. 
bioRxiv preprint doi: https://doi.org/10.1101/735563; this version posted August 15, 2019. The copyright holder for this preprint (which was not certified by peer review) is the author/funder, who has granted bioRxiv a license to display the preprint in perpetuity. It is made available under aCC-BY-NC-ND 4.0 International license.

I.C. Wagner et al.

A

placebo $<$ control partial correlations, $p<0.05$

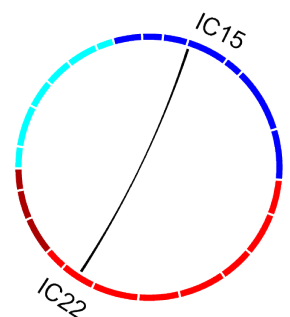

B

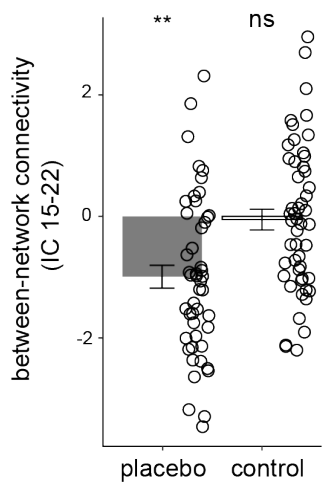

placebo control
D

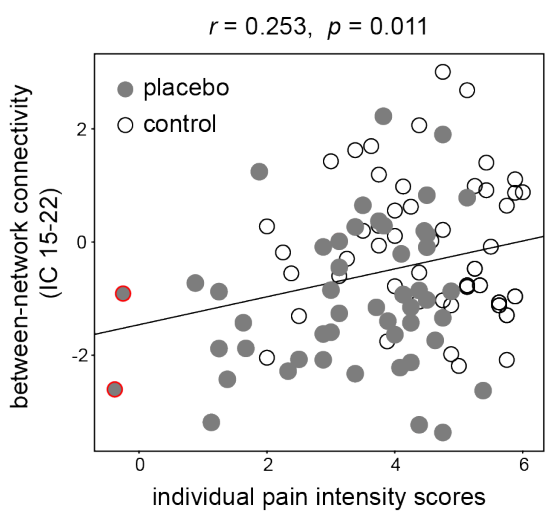

C

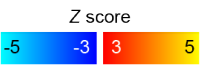

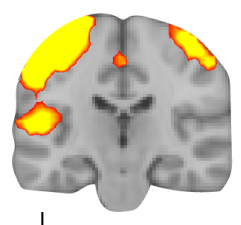

$\mathrm{L}$

$y=-22$

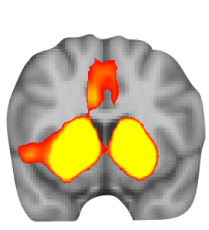

$y=10$

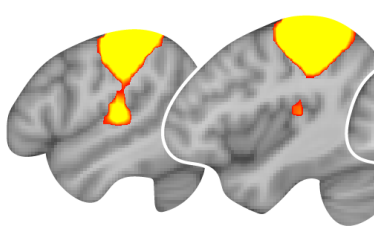

$x=-50$

$x=-40$

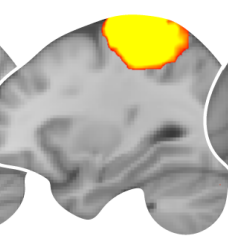

$x=-30$

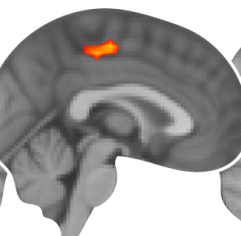

$x=0$

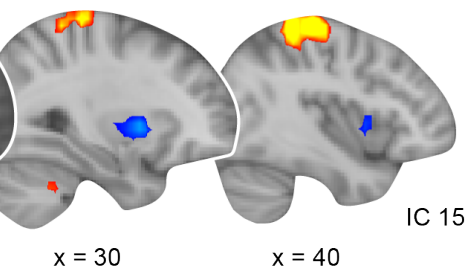

C 15

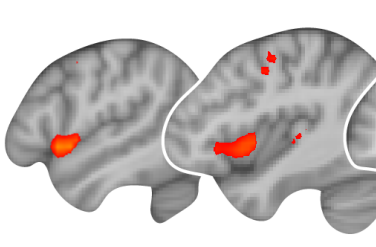

$x=-50$

$x=-40$

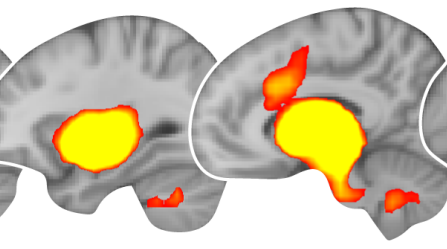

$x=-30$

$x=-10$

$x=30$

$x=40$

Fig. 4. Between-network connectivity differences between placebo and control groups. (A) Chord diagram visualizing the connectivity between independent component (IC) 15 and 22 was significantly different in placebo compared to control participants (placebo < control, $p=0.047$, corrected for multiple comparisons across all 625 network edges). (B) Between-network connectivity was negative in the placebo group $\left({ }^{* *}, p<0.001\right)$ but not significant (ns) in the control group. (C) IC 15 (upper panel, initially grouped together with cluster 2; Fig. 3A) and IC 22 (lower panel, initially grouped together with cluster 3; Fig. 3A). Coordinates of brain regions are described in Table 1. Brain slices are based on the mean structural scan of all participants. L, left. (D) Results showed a positive correlation of between-network connectivity (IC 15-22) with individual pain intensity scores across participants $(N=99)$. Thus, more negative coupling was associated with lower subjective pain intensity during the preceding pain task (Fig. 1CD). The correlation remained robust when removing two possible outliers from the placebo group (mean - 3 standard deviations, marked in red). 
Table 1. Networks IC 15 and 22. MNI coordinates represent the location of peak voxels. We report the first local maximum within each cluster. Percentages describe the probability of the peak voxel belonging to the labelled region. Anatomical nomenclature was obtained from the "Harvard-Oxford Cortical Structural Atlas", the "Harvard-Oxford Subcortical Structural Atlas", and the "Cerebellar Atlas in MNI152 space after normalization with FNIRT". IC, independent component; L, left; R, right.

\begin{tabular}{|c|c|c|c|c|c|}
\hline \multirow[b]{2}{*}{ ICs and brain regions } & \multicolumn{3}{|c|}{ MNI coordinates } & \multirow[b]{2}{*}{$z$-value } & \multirow[b]{2}{*}{ cluster size } \\
\hline & $x$ & $y$ & $z$ & & \\
\hline \multicolumn{6}{|l|}{ IC 15} \\
\hline 51\% Postcentral Gyrus, 5\% Precentral Gyrus, L & -46 & -22 & 56 & 17.1 & 941 \\
\hline $35 \%$ Postcentral Gyrus, $12 \%$ Superior Parietal Lobule, R & 42 & -38 & 64 & 7.04 & 210 \\
\hline Cerebellum, $\mathrm{R}$ & 18 & -50 & -32 & 6.03 & 90 \\
\hline $\begin{array}{l}60 \% \text { Precentral Gyrus, } 14 \% \text { Juxtapositional Lobule Cortex (formerly } \\
\text { Supplementary Motor Cortex), L }\end{array}$ & -2 & -18 & 52 & 5.13 & 37 \\
\hline Cerebellum, $58 \%$ R IX, 28\% Vermis VIIIb, R & 6 & -62 & -44 & 3.26 & 7 \\
\hline 6\% Temporal Occipital Fusiform Cortex, 5\% Occipital Fusiform Gyrus, L & -26 & -66 & -20 & 3.69 & 5 \\
\hline 1\% Lateral Occipital Cortex, inferior division, L & -30 & -82 & -28 & 3.26 & 4 \\
\hline 2\% Occipital Fusiform Gyrus, L & -42 & -74 & -28 & 3.15 & 1 \\
\hline \multicolumn{6}{|l|}{ IC 22} \\
\hline 91\% Thalamus, $\mathrm{L}$ & -14 & -10 & 8 & 18.5 & 2489 \\
\hline Cerebellum, $69 \%$ R Crus I, $25 \%$ R VI, R & 34 & -54 & -36 & 5.34 & 299 \\
\hline 34\% Precentral Gyrus, 2\% Middle Frontal Gyrus, L & -42 & -6 & 44 & 3.65 & 20 \\
\hline
\end{tabular}

\subsection{Between-network connectivity and relation to expectations in medication effectiveness}

To mitigate the effect of treatment expectations on resting-state fluctuations, we assessed connectivity after pain processing took place. However, treatment expectations might have still affected our results. We clarified this by testing the relationship of between-network connectivity and individual expectations in medication effectiveness assessed before (effectiveness $\mathrm{T}_{11}$ ) and after (effectiveness $\mathrm{S}_{\mathrm{T}}$ ) placebo conditioning in participants of the placebo group (Section 3.1.). Results showed no significant correlation of between-network connectivity with expectations in medication effectiveness rated before (effectiveness $\mathrm{T}_{1}: p=0.912$ ) or after placebo conditioning (effectiveness $2: p=0.364$ ). Thus, betweennetwork connectivity was not related to expectations in medication effectiveness in the placebo group.

\section{Discussion}

Effects of placebo analgesia on resting-state functional connectivity between networks have not yet been investigated so far. Here, we report first findings from an exploratory and predominantly data-driven analysis approach. We assessed the effects of placebo analgesia on network interactions during rest within a large-scale sample of healthy participants. Results showed significant inverse coupling between two networks that were centered on the bilateral somatosensory cortex (IC 15) and on the brainstem, thalamus, striatal regions, dorsal and rostral ACC, and the insula (IC 22) in placebo compared to control participants. The amount of inverse coupling was positively related to individual pain intensity scores across participants. In other words, more negative between-network connectivity was associated with lower subjective pain intensity during the preceding task. Results were unrelated to expectations of medication effectiveness in the placebo group. Our findings highlight how placebo analgesia affects intrinsic network fluctuations during rest. 
We hypothesized network interactions between the FPN/ECN, SN, and the sensory-motor network that should differ between placebo and control groups. Results partly confirmed our expectations as we found negative connectivity of a network that resembled the SN with a somatosensory network in placebo compared to control participants (Fig. 4). The classic SN consists of the dACC and insula, and is connected to subcortical regions such as the thalamus, (ventral) striatum, and the PAG (Seeley et al., 2007). The dACC was implicated in attention (Downar et al., 2000) and conflict monitoring (Botvinick et al., 2004), negative affect including anxiety and stress (Seeley et al., 2007; Hermans et al., 2011), pain perception (Downar et al., 2003; Legrain et al., 2011; Wager et al., 2013; Kucyi and Davis, 2015), but also reward-based decision making (Bush et al., 2002; Hayden and Platt, 2010) and positive affective processing (Bartels and Zeki, 2004). In a similar vein, the insula was associated with salience (Menon and Uddin, 2010), affective (Singer et al., 2009) and pain processing (Rodriguez-Raecke et al., 2010; Wiech et al., 2010), as well as interoception (Craig, 2002, 2009). Altogether, the SN was proposed to direct attention to relevant sensory input, to integrate sensory information with internal bodily states, and to prepare appropriate actions (Menon and Uddin, 2010; Gogolla, 2017).

Besides the common SN regions, the network we identified (IC 22) further comprised the rACC which is regarded as central for the down-regulation of subjective pain intensity during placebo analgesia (Wager and Atlas, 2015). Together with the PAG, the rACC is part of a descending circuit for pain modulation that further includes the rostral ventromedial medulla and its projections to the spinal cord (Fields, 2004). Previous studies using human neuroimaging showed placebo-related coupling between the rACC and PAG during pain processing (Petrovic et al., 2002; Bingel et al., 2006; Wager et al., 2007; Eippert et al., 2009), as well as increased connectivity of the $\mathrm{rACC}$ with the $\mathrm{SN}$ that predicted individual placebo responses at baseline (Sikora et al., 2016). Thus, interactions between the rACC and regions of the SN, including the PAG, appear crucial for placebo-induced pain relief.

The somatosensory network (IC 15) comprised the bilateral somatosensory cortex, supplemental motor area, and left posterior insula/superior temporal gyrus, and we propose that it potentially coded for the somatosensory representation of the noxious stimulation during the preceding task. Connectivity between IC 15 and 22 was negative in placebo compared to control participants such that increased activation in one network was related to decreased activation in the other and vice versa. We suggest that increased activation of brainstem, thalamus, striatal regions, dorsal and rostral ACC, and the insula (IC 22) underlies descending pain modulation after placebo which in turn might be associated with lower activation of somatosensory regions (IC 15). Across participants, the degree of inverse coupling between the networks was associated with lower individual pain intensity scores obtained during the preceding pain task (Fig. 4D), probing the behavioral relevance of these resting-state network fluctuations. This is in line with findings from Petrovic and colleagues (2002) who demonstrated similar engagement of rACC and brainstem in both placebo and opioid analgesia. Increased activation of brainstem, thalamus, striatal regions, dorsal and rostral ACC, and the insula (IC 22) might thus reflect placebo-induced, opioidergic activation related to the top-down modulation of pain (Baumgärtner et al., 2006; Eippert et al., 2009; Zunhammer et al., 2018). Most importantly, we found these connectivity changes during resting-state, in absence of actual pain anticipation or perception. Our findings thus suggest that placebo analgesia might affect communication between large-scale networks, potentially up-regulating opioidergic signaling even when pain is not present.

Network connectivity implies oscillatory synchrony between neuronal assemblies which is thought to facilitate information processing (Buzsaki and Draguhn, 2004). Certain brain networks, such as the FPN and the DMN, are often negatively correlated during resting-state (Fox et al., 2005; but see for example van Buuren et al., 2019) as well as during cognitively demanding tasks (Cole et al., 2012; but see Douw et al., 2016), whereby the magnitude of inverse coupling was linked to better task performance (Anticevic et al., 2012; Cole et al., 2012). Thus, negative between-network connectivity appears to affect information 
transfer in a functionally relevant manner. Fox and colleagues (2005) suggested that while positive connectivity might be involved in the integration of information across different brain regions, negative connectivity might segregate neuronal processes that subserve opposing goals. Negative coupling between the SN and somatosensory network might thus indicate increased activation of descending painmodulatory processes after placebo, segregating this network from somatosensory activation that might code for (preceding) noxious stimulation.

We did not find interactions of SN and somatosensory network with the FPN/ECN. A reason for this might be that we assessed resting-state functional connectivity after pain processing took place. Previous studies that investigated placebo effects on network connectivity focused on baseline resting-state periods in order to predict pain modulation (Kong et al., 2013) or individual placebo responses (Sikora et al., 2016; Tétreault et al., 2016). For instance, Kong and colleagues (2013) found increased resting-state coupling between the FPN/ECN and the rACC to be related to pain modulation in a subsequent task. The lateral prefrontal cortex is considered as central node of the FPN (Vincent et al., 2008; Yeo et al., 2011), important for cognitive control (Dosenbach et al., 2006, 2007; Cole et al., 2013) and the top-down modulation of pain (Wager et al., 2004, 2007; Watson et al., 2009; Petrovic et al., 2010). Previous results reporting FPN/ECN engagement were thus potentially related to treatment expectations or pain anticipation. We propose that our results are unrelated to such factors, as we measured resting-state fluctuations after pain processing took place. Furthermore, there was no significant relationship of between-network coupling and expectations in medication effectiveness across participants of the placebo group.

One possible explanation of our results could be that activation changes from the preceding task persisted into resting-state, thereby inducing the observed connectivity effects. If this was the case, we would assume comparable task-based activation changes, leading to significant connectivity between SN and somatosensory networks in both groups. Contrary to this, we did not find significant connectivity between the $\mathrm{SN}$ and somatosensory network in the control group (Fig. 4B). Thus, while participants showed inverse network coupling following placebo, we found network decoupling in the control group. Following this line of thought, several limitations need to be addressed. First, although we found significant differences in between-network connectivity between placebo and control groups, interpretation is limited by the between-subject nature of the study design. To further investigate placebo-induced changes in intrinsic network fluctuations, future studies should measure resting-state during placebo as well as control conditions in the same participants. Resting-state periods before and after placebo induction and pain processing might further be helpful to disentangle the effects of treatment expectations, pain anticipation and pain processing on network coupling, and to investigate trait characteristics that render individuals more or less susceptible to placebo-related belief inductions (Vachon-Presseau et al., 2018). Second, we found that inverse network coupling was positively related to individual pain intensity scores which we obtained from the preceding pain task (Fig. 1C). Future studies should use a more in-depth assessment of pain to generate individual pain intensity scores based on multiple tasks in order to replicate the current findings. However, in spite of these limitations, we provide first evidence for placebo-induced network changes during resting-state and their relation to behavioral pain modulation after pain processing.

\section{Conclusions}

To conclude, we investigated placebo effects on whole-brain resting-state functional connectivity in healthy human participants after pain processing. Our results revealed inverse coupling between two networks in placebo but not control participants. These networks were anchored on the bilateral somatosensory cortex, as well as on the brainstem, thalamus, striatal regions, dorsal and rostral ACC, and the anterior insula, respectively. Across participants, more negative between-network coupling was associated with lower individual pain intensity as assessed during a preceding pain task, but was unrelated 
to expectations of medication effectiveness in the placebo group. Altogether, these findings provide initial evidence that placebo analgesia affects the intrinsic communication between large-scale brain networks, even in the absence of pain. Our findings suggest that placebo analgesia increases activation within a descending pain-modulatory network, segregating it from somatosensory regions that might code for painful experiences.

\section{Conflict of Interest}

The authors declare no competing financial interests.

\section{Acknowledgements}

The authors would like to thank Daniel Graf, Bernadette Hippmann, Julia Hebestreit, Alexander Kudrna, and Andreas Martin (all University of Vienna) for assistance with functional MRI measurements; Fritz Zimprich (Medical University of Vienna) for medical support; and Andreas Gartus (University of Vienna) for technical support. The study was supported by the Vienna Science and Technology Fund (WWTF; Project CS11-016).

\section{References}

Abou-Elseoud A, Starck T, Remes J, Nikkinen J, Tervonen O, Kiviniemi V (2009) The effect of model order selection in group PICA. Hum Brain Mapp 31:1207-1216.

Anticevic A, Cole MW, Murray JD, Corlett PR, Wang X-J, Krystal JH (2012) The role of default network deactivation in cognition and disease. Trends Cogn Sci 16:584-592.

Bartels A, Zeki S (2004) The neural correlates of maternal and romantic love. Neuroimage 21:1155-1166.

Baumgärtner U, Buchholz H-G, Bellosevich A, Magerl W, Siessmeier T, Rolke R, Höhnemann S, Piel M, Rösch F, Wester H-J, Henriksen G, Stoeter P, Bartenstein P, Treede R-D, Schreckenberger M (2006) High opiate receptor binding potential in the human lateral pain system. Neuroimage 30:692-699.

Beckmann CF, DeLuca M, Devlin JT, Smith SM (2005) Investigations into resting-state connectivity using independent component analysis. Philos Trans R Soc B Biol Sci 360:1001-1013.

Beckmann CF, Smith SM (2004) Probabilistic Independent Component Analysis for Functional Magnetic Resonance Imaging. IEEE Trans Med Imaging 23:137-152.

Bingel U, Lorenz J, Schoell E, Weiller C, Büchel C (2006) Mechanisms of placebo analgesia: rACC recruitment of a subcortical antinociceptive network. Pain 120:8-15.

Bingel U, Wanigasekera V, Wiech K, Ni Mhuircheartaigh R, Lee MC, Ploner M, Tracey I (2011) The Effect of Treatment Expectation on Drug Efficacy: Imaging the Analgesic Benefit of the Opioid Remifentanil. Sci Transl Med 3:70ra14-70ra14.

Botvinick MM, Cohen JD, Carter CS (2004) Conflict monitoring and anterior cingulate cortex: an update. Rev TRENDS Cogn Sci 8:539-546.

Bush G, Vogt BA, Holmes J, Dale AM, Greve D, Jenike MA, Rosen BR (2002) Dorsal anterior cingulate cortex: a role in reward-based decision making. Proc Natl Acad Sci U S A 99:523-528.

Buzsaki G, Draguhn A (2004) Neuronal Oscillations in Cortical Networks. Science (80- ) 304:1926-1929.

Cole MW, Reynolds JR, Power JD, Repovs G, Anticevic A, Braver TS (2013) Multi-task connectivity reveals flexible hubs for adaptive task control. Nat Neurosci 16:1348-1355.

Cole MW, Yarkoni T, Repovs G, Anticevic A, Braver TS (2012) Global connectivity of prefrontal cortex predicts cognitive control and intelligence. J Neurosci 32:8988-8999. 
Craig AD (2002) How do you feel? Interoception: the sense of the physiological condition of the body. Nat Rev Neurosci 3:655-666.

Craig AD (2009) How do you feel - now? The anterior insula and human awareness. Nat Rev Neurosci 10:59-70.

Damoiseaux JS, Rombouts SARB, Barkhof F, Scheltens P, Stam CJ, Smith SM, Beckmann CF (2006) Consistent resting-state networks across healthy subjects. Proc Natl Acad Sci 103:13848-13853.

Dosenbach NUF, Fair DA, Miezin FM, Cohen AL, Wenger KK, Dosenbach RAT, Fox MD, Snyder AZ, Vincent JL, Raichle ME, Schlaggar BL, Petersen SE (2007) Distinct brain networks for adaptive and stable task control in humans. Proc Natl Acad Sci 104:11073-11078.

Dosenbach NUF, Visscher KM, Palmer ED, Miezin FM, Wenger KK, Kang HC, Burgund ED, Grimes AL, Schlaggar BL, Petersen SE (2006) A Core System for the Implementation of Task Sets. Neuron 50:799812.

Douw L, Wakeman DG, Tanaka N, Liu H, Stufflebeam SM (2016) State-dependent variability of dynamic functional connectivity between frontoparietal and default networks relates to cognitive flexibility. Neuroscience 339:12-21.

Downar J, Crawley AP, Mikulis DJ, Davis KD (2000) A multimodal cortical network for the detection of changes in the sensory environment. Nat Neurosci 3:277-283.

Downar J, Mikulis DJ, Davis KD (2003) Neural correlates of the prolonged salience of painful stimulation. Neuroimage 20:1540-1551.

Eippert F, Bingel U, Schoell ED, Yacubian J, Klinger R, Lorenz J, Büchel C (2009) Activation of the Opioidergic Descending Pain Control System Underlies Placebo Analgesia. Neuron 63:533-543.

Fields H (2004) State-dependent opioid control of pain. Nat Rev Neurosci 5:565-575.

Fox MD, Snyder AZ, Vincent JL, Corbetta M, Van Essen DC, Raichle ME (2005) The human brain is intrinsically organized into dynamic, anticorrelated functional networks. Proc Natl Acad Sci 102:9673-9678.

Geuter S, Eippert F, Hindi Attar C, Büchel C (2013) Cortical and subcortical responses to high and low effective placebo treatments. Neuroimage 67:227-236.

Gogolla N (2017) The insular cortex. Curr Biol 27:PR580-R586.

Griffanti L, Douaud G, Bijsterbosch J, Evangelisti S, Alfaro-Almagro F, Glasser MF, Duff EP, Fitzgibbon S, Westphal R, Carone D, Beckmann CF, Smith SM (2017) Hand classification of FMRI ICA noise components. Neuroimage 154:188-205.

Hayden BY, Platt ML (2010) Neurons in anterior cingulate cortex multiplex information about reward and action. J Neurosci 30:3339-3346.

Hermans EJ, van Marle HJF, Ossewaarde L, Henckens MJAG, Qin S, van Kesteren MTR, Schoots VC, Cousijn H, Rijpkema M, Oostenveld R, Fernandez G (2011) Stress-Related Noradrenergic Activity Prompts Large-Scale Neural Network Reconfiguration. Science (80- ) 334:1151-1153.

Jenkinson M, Beckmann CF, Behrens TEJ, Woolrich MW, Smith SM (2012) FSL. Neuroimage 62:782-790.

Kong J, Jensen K, Loiotile R, Cheetham A, Wey H-Y, Tan Y, Rosen B, Smoller JW, Kaptchuk TJ, Gollub RL (2013) Functional connectivity of the frontoparietal network predicts cognitive modulation of pain. Pain 154:459-467.

Kucyi A, Davis KD (2015) The dynamic pain connectome. Trends Neurosci 38:86-95.

Legrain V, lannetti GD, Plaghki L, Mouraux A (2011) The pain matrix reloaded. Prog Neurobiol 93:111-124.

Lorenz J, Minoshima S, Casey KL (2003) Keeping pain out of mind: the role of the dorsolateral prefrontal 
I.C. Wagner et al.

cortex in pain modulation. Brain 126:1079-1091.

Menon V, Uddin LQ (2010) Saliency, switching, attention and control: a network model of insula function. Brain Struct Funct 214:655-667.

Petrovic P, Kalso E, Petersson KM, Andersson J, Fransson P, Ingvar M (2010) A prefrontal non-opioid mechanism in placebo analgesia. Pain 150:59-65.

Petrovic P, Kalso E, Petersson KM, Ingvar M (2002) Placebo and Opioid Analgesia-- Imaging a Shared Neuronal Network. Science (80- ) 295:1737-1740.

Power JD, Barnes KA, Snyder AZ, Schlaggar BL, Petersen SE (2012) Spurious but systematic correlations in functional connectivity MRI networks arise from subject motion. Neuroimage 59:2142-2154.

Price DD, Finniss DG, Benedetti F (2008) A Comprehensive Review of the Placebo Effect: Recent Advances and Current Thought. Annu Rev Psychol 59:565-590.

Pruim RHR, Mennes M, Buitelaar JK, Beckmann CF (2015a) Evaluation of ICA-AROMA and alternative strategies for motion artifact removal in resting state fMRI. Neuroimage 112:278-287.

Pruim RHR, Mennes M, van Rooij D, Llera A, Buitelaar JK, Beckmann CF (2015b) ICA-AROMA: A robust ICAbased strategy for removing motion artifacts from fMRI data. Neuroimage 112:267-277.

Rodriguez-Raecke R, Doganci B, Breimhorst M, Stankewitz A, Buchel C, Birklein F, May A (2010) Insular Cortex Activity Is Associated with Effects of Negative Expectation on Nociceptive Long-Term Habituation. J Neurosci 30:11363-11368.

Rütgen M, Seidel E-M, Silani G, Riečanský I, Hummer A, Windischberger C, Petrovic P, Lamm C (2015) Placebo analgesia and its opioidergic regulation suggest that empathy for pain is grounded in self pain. Proc Natl Acad Sci 112:E5638-E5646.

Scott DJ, Stohler CS, Egnatuk CM, Wang H, Koeppe RA, Zubieta J-K (2007) Individual differences in reward responding explain placebo-induced expectations and effects. Neuron 55:325-336.

Seeley WW, Menon V, Schatzberg AF, Keller J, Glover GH, Kenna H, Reiss AL, Greicius MD (2007) Dissociable intrinsic connectivity networks for salience processing and executive control. J Neurosci 27:2349-2356.

Shirer WR, Jiang H, Price CM, Ng B, Greicius MD (2015) Optimization of rs-fMRI Pre-processing for Enhanced Signal-Noise Separation, Test-Retest Reliability, and Group Discrimination. Neuroimage 117:67-79.

Sikora M, Heffernan J, Avery ET, Mickey BJ, Zubieta J-K, Peciña M (2016) Salience Network Functional Connectivity Predicts Placebo Effects in Major Depression. Biol psychiatry Cogn Neurosci neuroimaging 1:68-76.

Singer T, Critchley HD, Preuschoff K (2009) A common role of insula in feelings, empathy and uncertainty. Trends Cogn Sci 13:334-340.

Smith SM, Vidaurre D, Beckmann CF, Glasser MF, Jenkinson M, Miller KL, Nichols TE, Robinson EC, SalimiKhorshidi G, Woolrich MW, Barch DM, Uğurbil K, Van Essen DC (2013) Functional connectomics from resting-state fMRI. Trends Cogn Sci 17:666-682.

Smith D V, Utevsky A V, Bland AR, Clement N, Clithero JA, Harsch AEW, McKell Carter R, Huettel SA (2014) Characterizing individual differences in functional connectivity using dual-regression and seed-based approaches. Neuroimage 95:1-12.

Tétreault P, Mansour A, Vachon-Presseau E, Schnitzer TJ, Apkarian AV, Baliki MN (2016) Brain Connectivity Predicts Placebo Response across Chronic Pain Clinical Trials. PLOS Biol 14:e1002570.

Vachon-Presseau E, Berger SE, Abdullah TB, Huang L, Cecchi GA, Griffith JW, Schnitzer TJ, Apkarian AV (2018) Brain and psychological determinants of placebo pill response in chronic pain patients. Nat 
I.C. Wagner et al.

Commun 9:3397.

van Buuren M, Wagner IC, Fernández G (2019) Functional network interactions at rest underlie individual differences in memory ability. Learn Mem 26:9-19.

Vincent JL, Kahn I, Snyder AZ, Raichle ME, Buckner RL (2008) Evidence for a Frontoparietal Control System Revealed by Intrinsic Functional Connectivity. J Neurophysiol 100:3328-3342.

Wager TD, Atlas LY (2015) The neuroscience of placebo effects: connecting context, learning and health. Nat Rev Neurosci 16:403-418.

Wager TD, Atlas LY, Lindquist MA, Roy M, Woo C-W, Kross E (2013) An fMRI-Based Neurologic Signature of Physical Pain. N Engl J Med 368:1388-1397.

Wager TD, Rilling JK, Smith EE, Sokolik A, Casey KL, Davidson RJ, Kosslyn SM, Rose RM, Cohen JD (2004) Placebo-Induced Changes in $\mathrm{FMRI}$ in the Anticipation and Experience of Pain. Science (80- ) 303:1162-1167.

Wager TD, Scott DJ, Zubieta J-K (2007) Placebo effects on human mu-opioid activity during pain. Proc Natl Acad Sci 104:11056-11061.

Ward JH (1963) Hierarchical Grouping to Optimize an Objective Function. J Am Stat Assoc 58:236-244.

Watson A, El-Deredy W, lannetti GD, Lloyd D, Tracey I, Vogt BA, Nadeau V, Jones AKP (2009) Placebo conditioning and placebo analgesia modulate a common brain network during pain anticipation and perception. PAIN ${ }^{\circledR}$ 145:24-30.

Wiech K, Lin C-s., Brodersen KH, Bingel U, Ploner M, Tracey I (2010) Anterior Insula Integrates Information about Salience into Perceptual Decisions about Pain. J Neurosci 30:16324-16331.

Yeo BT, Krienen FM, Sepulcre J, Sabuncu MR, Lashkari D, Hollinshead M, Roffman JL, Smoller JW, Zöllei L, Polimeni JR, Fischl B, Liu H, Buckner RL (2011) The organization of the human cerebral cortex estimated by intrinsic functional connectivity. J Neurophysiol 106:1125-1165.

Zubieta J-K, Bueller JA, Jackson LR, Scott DJ, Xu Y, Koeppe RA, Nichols TE, Stohler CS (2005) Placebo Effects Mediated by Endogenous Opioid Activity on Mu-Opioid Receptors. J Neurosci 25:7754-7762.

Zubieta J-K, Smith YR, Bueller JA, Xu Y, Kilbourn MR, Jewett DM, Meyer CR, Koeppe RA, Stohler CS (2001) Regional Mu Opioid Receptor Regulation of Sensory and Affective Dimensions of Pain. Science (80-) 293:311-315.

Zunhammer M, Bingel U, Wager TD, Placebo Imaging Consortium (2018) Placebo Effects on the Neurologic Pain Signature. JAMA Neurol 75:1321. 